\title{
Design of Wireless Smart Sensor Module for Infant Incubator Test
}

\author{
Guoguang $\mathrm{Wu}^{1, \mathrm{a}}$, Shangwen $\mathrm{Chen}^{2, \mathrm{~b}}$ \\ ${ }^{1}$ Guangdong Provincial Institute of Metrology, Guangzhou, China \\ ${ }^{2}$ Guangdong Provincial Institute of Metrology, Guangzhou, China \\ aimguangzi@163.com, ${ }^{b}$ cswclx@163.com
}

Keywords: Infant Incubator; IEEE 1451; ZigBee; Smart Sensor.

Abstract: Aiming to solve the problem of difficulty of wiring and operation of infant incubator test, we put forward a wireless smart sensor module based on IEEE 1451. The module can realize the function of self recognition, plug and play. The key technology of wireless smart sensor module is thoroughly discussed, and the circuit module is developed. The test indicates that the module can be used in the detection of infant incubator.

\section{Introduction}

Infant incubator is for premature, low birth weight infants and critically ill infants with similar maternal intrauterine environment equipment. It must be tested periodically to make sure the safety performance to the infant patient ${ }^{[1]}$. While currently commercial test products are mainly wired, or not convenient to use. Such as the Datrend Company developed the Incutest, FLUKE Company developed the INCU, National Institute of Measurement and Testing Technology developed the CDBI-3000, and Shenzhen Company developed the SJ90. In order to improve the work efficiency, a new module with wireless smart sensor is designed with the IEEE1451 protocol. The IEEE 1451 is a new universal intelligent sensor interface standard, proposed by Kang Lee and other scholars in 1993. With standard data structure and communication protocol, so that the sensor can realize self identification, the "plug and play" and other functions ${ }^{[2-4]}$. The IEEE 1451 protocol are widely used in military, environmental monitoring, medical and other fields in recent years ${ }^{[5-7]}$. This paper will elaborate the principle, implementation of the wireless smart sensor module for infant incubator test.

\section{Block diagram of Wireless Smart Sensor Module}

Fig. 1 is the block diagram of smart wireless sensor platform based on IEEE 1451.5 for infant incubator test. The platform mainly composed of two modules. The WTIM (Wireless Transducer Interface Module) and NCAP (Network capable Application Processor), and communicate with ZigBee wireless protocol. WTIM is the consisted of temperature sensor Tsic 716, humidity sensor SHT 75, power module Max1797 and wireless chip JN5139. JN5139 is a low power ZigBee wireless module, the operating voltage is (2.7 3.6) voltage, In order to achieve low power consumption, WTIM is powered by two section 7 battery. While the power supply of Tsic 716 is 5 voltage which to ensure the accuracy of the sensor, we must carry out level conversion. MAX1797 is a boost DC-DC converter, the 3 voltage is converted to 5 voltage to supply Tsic 716 .

\section{Key Functional Module Design}

The key technology of wireless smart sensor for infant incubator are relative humidity data reading, temperature data reading, WTIM TEDS design, ZigBee wireless protocol design.

Relative humidity data reading

SHT 75 is a temperature and relative humidity sensor, but the temperature accuracy is $\pm 0.3^{\circ} \mathrm{C}$, it 


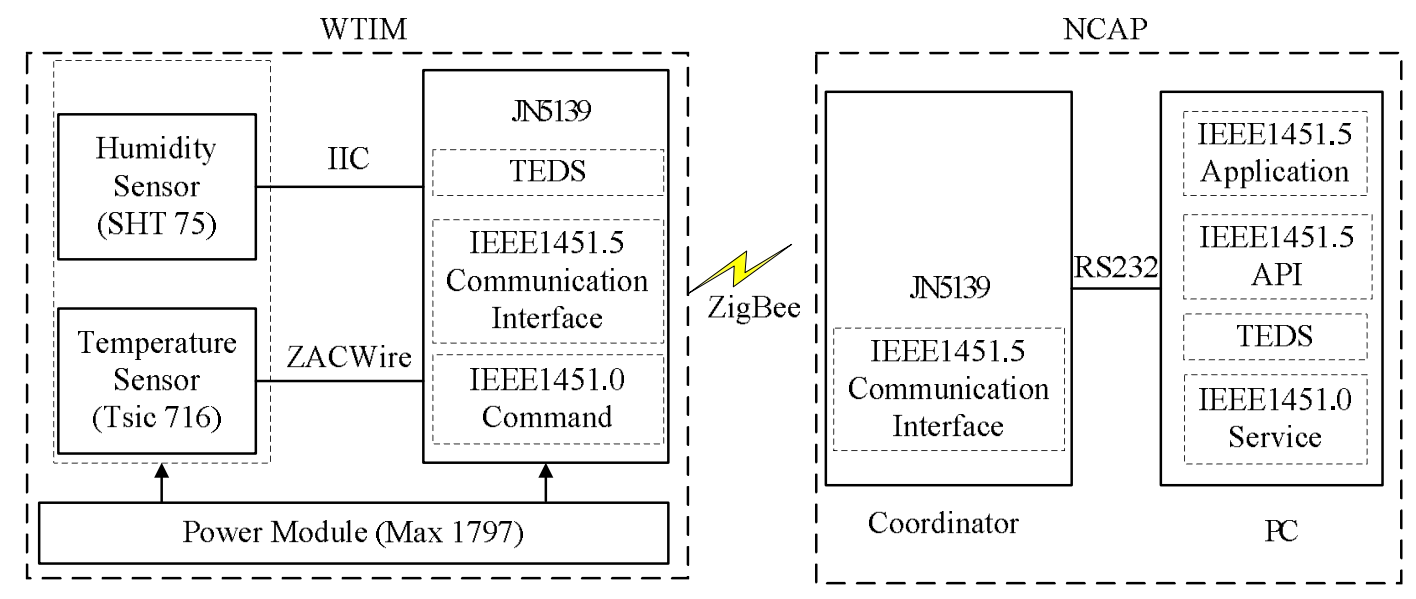

Fig. 1 The block diagram of smart wireless smart sensor platform based on IEEE 1451.5

can not meet the requirement $\pm 0.2^{\circ} \mathrm{C}$ of document JJF $1260-2010$. So we only use the relative humidity data. The relative humidity data can be used in two modes, 12 bit or 8 bit. As the sensor is non-linier, and the real temperature and the reference temperature is not the same, the data should be compensated by linear and temperature. The compensation equation is in Equation 1, 2, where $\mathrm{SO}_{\mathrm{RH}}$ is the output coded data.

$$
\begin{aligned}
& \mathrm{RH}_{\text {linear }}=\mathrm{C}_{1}+\mathrm{C}_{2} \cdot \mathrm{SO}_{\mathrm{RH}}+\mathrm{C}_{3} \cdot \mathrm{SO}_{\mathrm{RH}}{ }^{2} \quad[\% \mathrm{RH}] \\
& \mathrm{RH}_{\text {true }}=\left(\mathrm{T}_{{ }^{\circ} \mathrm{C}}-25\right) \cdot\left(\mathrm{t}_{1}+\mathrm{t}_{2} \cdot \mathrm{SO}_{\mathrm{RH}}\right)+\mathrm{RH}_{\text {linear }} \quad[\% \mathrm{RH}]
\end{aligned}
$$

Table 1 Compensation coefficients for linear and temperature

\begin{tabular}{rccccc}
\hline $\mathrm{SO}_{\mathrm{RH}}$ & $\mathrm{C}_{1}$ & $\mathrm{C}_{2}$ & $\mathrm{C}_{3}$ & $\mathrm{t}_{1}$ & $\mathrm{t}_{2}$ \\
\hline 12 bit & -2.0468 & 0.0367 & $-1.5955 \mathrm{E}-6$ & 0.01 & 0.00008 \\
8 bit & -2.0468 & 0.5872 & $-4.0845 \mathrm{E}-4$ & 0.01 & 0.00128 \\
\hline
\end{tabular}

Temperature data reading

Tsic 716 is a ZACWire single bus interface, 14 bit data output digital temperature sensor. Under the 5 voltage power supply condition, the measurement accuracy is up to $\pm 0.07^{\circ} \mathrm{C}$. It meets the requirement of document JJF 1260-2010 which demand the maximum allowable error is $\pm 0.2^{\circ} \mathrm{C}$. The measuring range is $-(10 \sim 60)^{\circ} \mathrm{C}$. The temperature data must be transmitted through two bytes. Fig. 2 is the ZAcwire bit encoding format. The start bit is $50 \%$ duty cycle, which is used to set the encoding bit judgment time, the logic "1" is the $75 \%$ duty cycle, and the logic " $0 "$ is the $25 \%$ duty

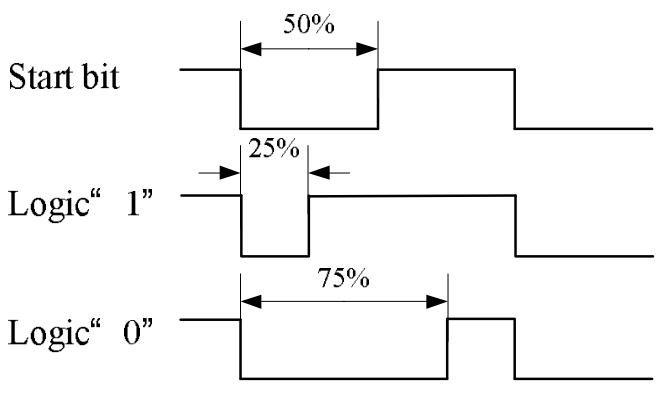

Fig. 2 Bit codint of the temperature sensor data cycle. The temperature is calculated in Equaton 3.

$$
T=\frac{T_{14}}{16384} \times(\mathrm{HT}-\mathrm{LT})+\mathrm{LT} \quad\left[{ }^{\circ} \mathrm{C}\right]
$$

Where $T_{14}$ is the output digital data, HT is high temperature threshold value $60, \mathrm{LT}$ is the low temperature threshold value -10 . 


\section{WTIM TEDS design}

WTIM uses JN5139 as a micro controller which integrated 192kB ROM, rich in IO interface and wireless communication unit. TEDS (Transducer Electronic Data sheet) is an important step to achieve communications between WTIM and NCAP. It consists of several kinds of TEDS, like Meta TEDS, Channel TEDS, and Physics TEDS which describe the total information, channel properties, and physics character. TEDS provides the necessary conditions for the realization of "plug and play" in the wireless sensor interface. Usually TEDS is saved in the MCU, when WTIM is powered and ask to connect to NCAP, the TEDS is sent to the NCAP to complete the transmitter with self describing process, which reflects the characteristics of WTIM self identification, and "plug and play". The most important TEDS are Meta TEDS, Channel TEDS, and Physics TEDS. Meta TEDS is used to identify the WTIM module location, manufacturer, the realization of the transmitter channel number and other information. Channel TEDS is mainly used for each channel function model, physical units, sampling cycle, measuring upper and lower limits. IEEE1451.5 consists of Physics TEDS, defining a range of wireless communication parameters. TEDS used in WTIM are shown in Table 1.

Table 1 The main TEDS structure of WTIM

\begin{tabular}{lll|lll}
\hline & \multicolumn{2}{c|}{ Meta TEDS } & \multicolumn{3}{c}{ Transducer } \\
\hline Symbol & Type & Describition & Symbol & Type & Describition \\
\hline TEDSlength & UInt32 & TEDS length & TEDSlength & UInt32 & TEDS length \\
TEDSID & UInt8 & TEDS sign & TEDSID & UInt8 & TEDS sign \\
UUID & UUID & $\begin{array}{l}\text { Global uniquer } \\
\text { indentification }\end{array}$ & LowLimit & Float32 & Measuring lower limit \\
OholdOff & Float32 & Operation overtime & HiLimit & Float32 & Measuring upper limit \\
TestTime & Float32 & Self test time & SUints & Uints & Unit \\
MaxChan & UInt16 & Channel numbers & SPeriod & Float32 & Sample period \\
\hline
\end{tabular}

ZigBee wireless communication protocol design 
ZigBee is a short distance, low power consumption, local area network protocol based on IEEE 802.15.4 standard. In IEEE 1451 protocol, ZigBee is one of optional wireless standards. We use JN5139 to accomplish the ZigBee wireless communication between NCAP and WTIM. Fig. 3 is the flow chart of WTIM and NCAP. When powered up, NCAP acts as a coordinator, and searches if the PAN network with the same ID is existed. If not, it starts to establish wireless ZigBee network and wait for WTIM module to connect. When WTIM powered up, it acts as an end device, and access to coordinator. NCAP distributes the network parameters to WTIM, like PAN ID, channel number. When the network is joined, NCAP will send read TEDS command to get the message of joined WTIM, so as to get the sensor type, data format, and other parameters. In normal data transformation mode, WTIM would periodically read the temperature data and relative humidity from Tsic 716 for and SHT 75, and send to NCAP. NCAP receives and decodes the data, and displays in PC.

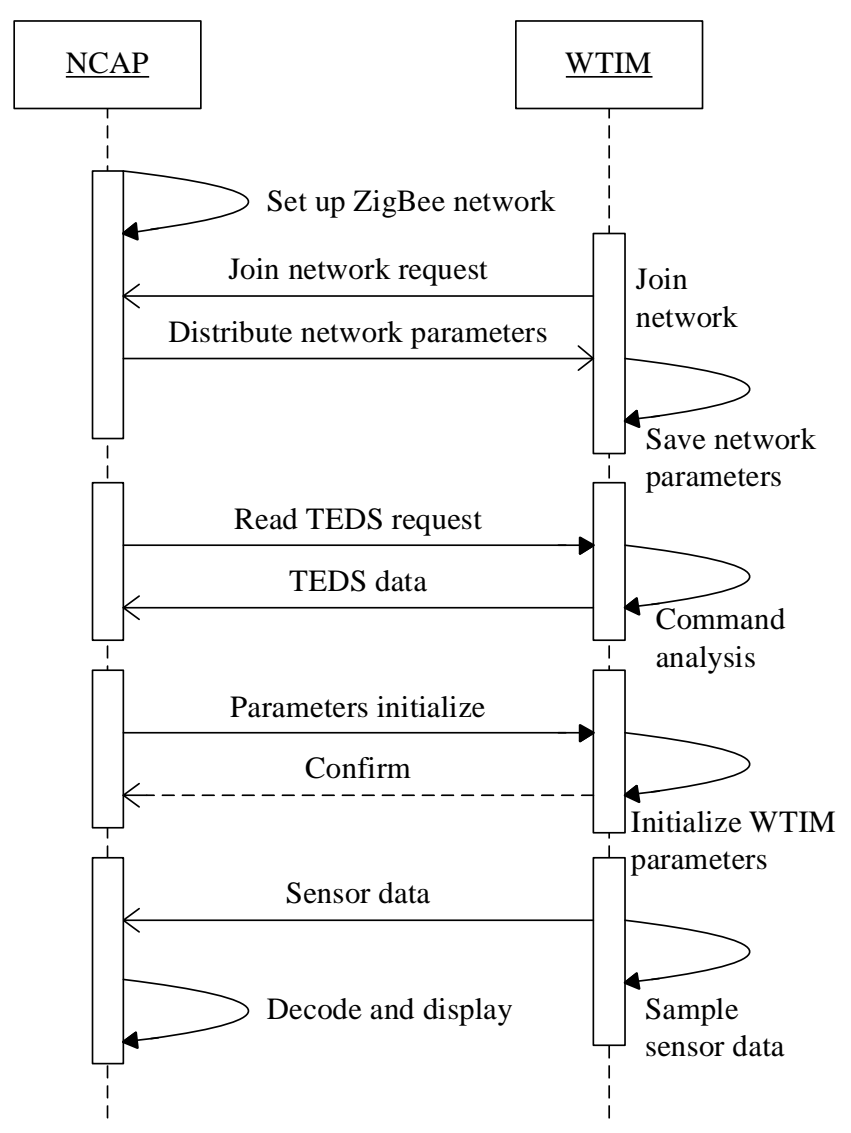

Fig. 3 Program flow chart of WTIM and NCAP

\section{Test and Implementation}

Fig. 4 is the diagram of smart wireless smart sensor board, the temperature and humidity sensors are plug in the interface which can be replaced conveniently when in damage. After long time test, the circuit is proved to work smoothly, and meets the requirement for test the infant incubator.

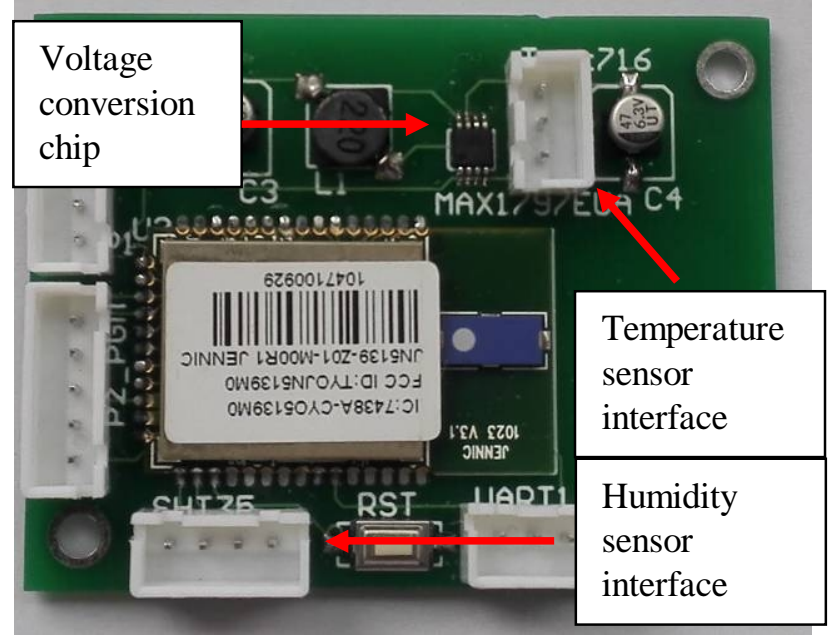

(a) Wireless Sensor board

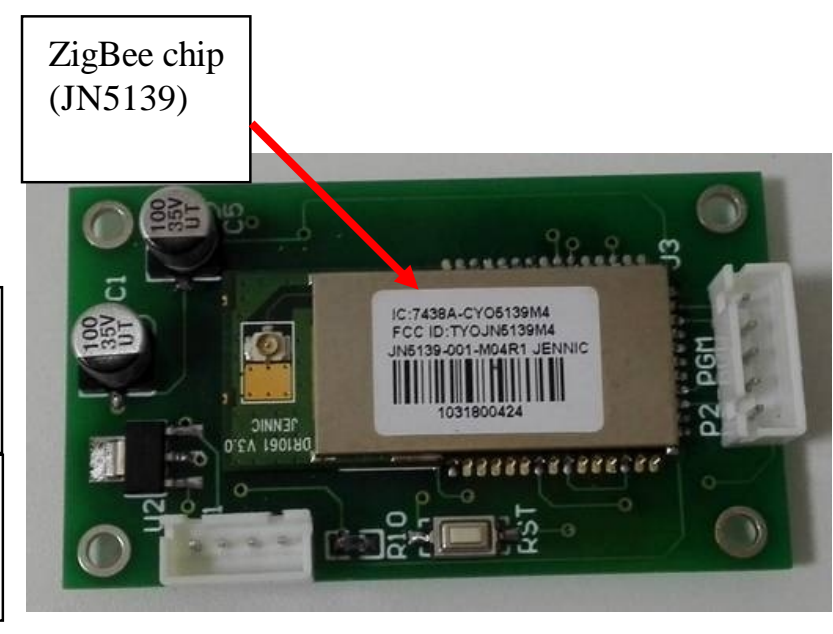

ZigBee wireless receiving board

Fig. 4 The diagram of smart wireless smart sensor board

Fig. 5 is the software interface when testing the infant incubator. We place five wireless sensor boards in the infant incubator. The data is transmitted once per second. 


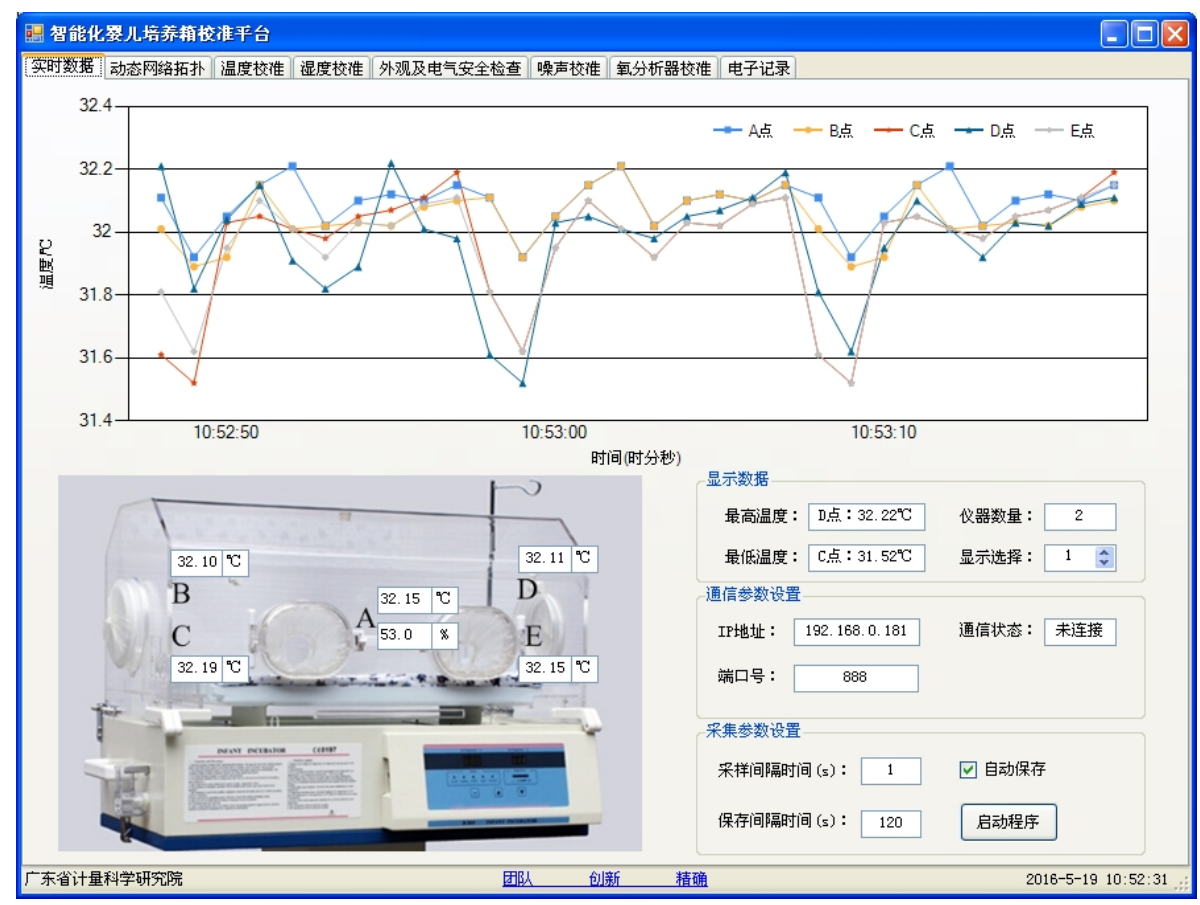

Fig. 5 The software interface

\section{Conclusions}

This paper implements a wireless smart sensor module for infant incubator test based on IEEE 1451. The module can meet the technical requirements of the current document. The advantage of compact, intellengent, make it easily to use which improve the efficiency of testing infant incubator, and has good application value for other applications.

\section{Acknowledgements}

This work was financially supported by the Science and Technology Project of Administration of Quality and Technology Supervision of Guangdong Province (2015CJ01).

\section{References}

[1] Mo Li-ming. Practice and Technology Discussion of Infant Incubator Quality Control[J]. China Medical Devices. 2011, 26(3): 49-51.

[2] D. Wobschall. Networked sensor monitoring using the universal IEEE 1451 Standard [J]. Instrumentation \& Measurement Magazine, IEEE, 2008, 11(2): 18-22.

[3] L. Bissi, P. Placidi, A. Scorzoni, et al. Environmental monitoring system compliant with the IEEE 1451 standard and featuring a simplified transducer interface [J]. Sensors and Actuators A: Physical, 2007, 137(1): 175-184.

[4] L. WEN-yi, H. Zhi-jun. Hardware design of intellignetial sensor based on IEEE1451.4 standard interface [J]. Electronic Design Engineering, 2009, 17(3): 106-110.

[5] M. Lee, T. M. Gatton. Wireless Health Data Exchange for Home Healthcare Monitoring Systems[J]. Sensors, 2010, 10(4): 3243-3260.

[6] A. Kumar, I. P. Singh, S. K. Sud. Energy Efficient and Low-Cost Indoor Environment Monitoring System Based on the IEEE 1451 Standard [J]. Sensors Journal, IEEE, 2011, 11(10): 2598-2610. 
[7] N. Kularatna, B. H. Sudantha. An Environmental Air Pollution Monitoring System Based on the IEEE 1451 Standard for Low Cost Requirements [J]. Sensors Journal, IEEE, 2008, 8(4): 415-422. 